\title{
Segmentation of Polarimetric SAR Data using Contour Information via Spectral Graph Partitioning
}

\author{
Kaan Ersahin, Ian G. Cumming and Rabab K. Ward \\ Department of Electrical and Computer Engineering \\ The University of British Columbia \\ Vancouver, B.C., CANADA
}

\begin{abstract}
A new method for segmenting polarimetric Synthetic Aperture Radar (POLSAR) data is proposed. Image segmentation is formulated as a graph partitioning problem. Spectral graph partitioning - known to provide perceptually plausible image segmentation results using one or more cues (e.g., similarity, proximity, contour continuity) - is applied on POLSAR image data. The degree of similarities between pairs of pixels are calculated based on contour information. Graph partitioning is performed using the Multiclass Spectral Clustering method that minimizes the normalized cut cost function to ensure minimal similarity between partitions. The resulting segmentation is an approximation to the global optimal solution. C-band POLSAR data acquired by CV-580 are used for testing the performance. The results are found to closely agree with manual segmentations.
\end{abstract}

\section{INTRODUCTION}

Spaceborne Synthetic Aperture Radar (SAR) systems with polarimetric modes are among the most important tools used in today's remote sensing tasks. A number of satellite systems are already operational or soon will be (e.g., ENVISAT, ALOS, TerraSAR-X, RADARSAT-2). Large volumes of data need to be analysed and interpreted on a daily basis, thus automated procedures are required. Segmentation is an important initial step for data interpretation and is a very challenging task to automate. However, visual interpretation of SAR image data by human experts is relatively easy, yet time consuming. Established techniques for polarimetric SAR data analysis (e.g., polarimetric decomposition [1], Wishart classifier and its variants [2], [3]) have the following shortcoming - they do not utilize the visual content and global information available in image data. The main focus of our work is to utilize some of this information that helps humans perform image segmentation so well.

For many problems in computer vision, the ultimate goal is to reach the performance level of the human vision system (HVS). This may be possible through a good understanding of how humans handle the task. For humans, an image represents more than a collection of pixels: it is a meaningful organization of objects or patterns. In the 1930s, Gestalt psychologists studied this important phenomenon - perceptual organization - and reported several factors that contribute to this process: similarity, proximity, contour continuity, symmetry and closure. These are known as cues in the psychology literature.

Over the last few decades, research in computer vision has sought methodologies that can utilize these ideas for solving grouping problems. Recently, a promising technique based on pairwise affinities (i.e., similarities) has emerged. The degree of similarity between pairs of data points is encoded in a pairwise affinity matrix and the grouping task is performed using spectral graph partitioning [4], [5]. This approach allows contributions from multiple cues like the HVS does, and offers a way to obtain a globally optimal solution. Therefore, segmentation results that are perceptually plausible (i.e., consistent with how humans perceive) can be produced. This makes spectral graph partitioning a promising candidate for automated analysis and interpretation of SAR data.

Ersahin et al. [6] used this technique on polarimetric SAR images for the first time, where the affinity matrix was formed using patch-based similarity and proximity between pairs of pixels. In this paper, we take a different approach to compute the pairwise affinities. Contour information (i.e., directional image features such as field boundaries) is utilized via orientation energy. Preliminary results obtained using the proposed scheme are found to be more homogenous than the Wishart classifier output, and they closely agree with the manual segmentations. Detailed analysis that compares the performance to state-of-the-art techniques is underway.

\section{Spectral Graph Partitioning}

Both clustering and image segmentation can be formulated as a graph partitioning problem. This is achieved by representing a set of points in an arbitrary feature space using an undirected graph $G=\{V, E\}$, where $V$ and $E$ are the nodes and the edges, respectively. Each node on the graph corresponds to a data point and an edge between two nodes, $u$ and $v$, is associated with a weight, $\omega(u, v)$, that indicates the similarity between $u$ and $v$ according to some criterion. In general, $G$ is a fully connected graph (i.e., each node is connected to all the other nodes). For bi-partitioning such a graph, it is intuitive to minimize the similarity of candidate partitions (i.e., cut). $\operatorname{cut}\left(V_{1}, V_{2}\right)$ represents the similarity of candidate partitions $V_{1}$ and $V_{2}$. It is quantified by the sum of the weights between the nodes in $V_{1}$ and the nodes in $V_{2}$. However, minimizing the cut favors partitions with isolated nodes. This bias can be avoided by minimizing the normalized cut (ncut):

$$
\operatorname{ncut}\left(V_{1}, V_{2}\right)=\frac{\operatorname{cut}\left(V_{1}, V_{2}\right)}{\operatorname{assoc}\left(V_{1}, V\right)}+\frac{\operatorname{cut}\left(V_{1}, V_{2}\right)}{\operatorname{assoc}\left(V_{2}, V\right)}
$$

where $\operatorname{assoc}\left(V_{1}, V\right)$ is the sum of the weights between the nodes in $V_{1}$ and all the nodes in the graph, $V$. 
Shi and Malik [4] showed that finding a global minimum for the ncut cost function can be approximated by solving the generalized eigenvalue system for the normalized graph Laplacian:

$$
\mathcal{L}=D^{-\frac{1}{2}}(D-W) D^{-\frac{1}{2}}
$$

where $W$ is the $N \times N$ matrix whose entries are the weights, $\omega(i, j)$; and $D$ is a diagonal matrix whose elements are the sum of the rows of $W$. To bi-partition the graph, the eigenvector that corresponds to the second smallest eigenvalue is used. This approach has also been applied recursively to obtain more than two partitions [4].

$\mathrm{Ng}$ et al. [5] extended this technique to the k-way partitioning case, however they used a slightly different notation. The affinity matrix $A$ is used instead of the similarity matrix $W$ and $I-\mathcal{L}$ (where $I$ is the identity matrix) is replaced with the normalized affinity matrix:

$$
L=D^{-\frac{1}{2}} A D^{-\frac{1}{2}}
$$

Both techniques start by collecting local evidence on how likely it is that two data points belong to the same partition. This is achieved by forming a matrix whose entries represent pairwise similarities defined appropriately. Later, a global decision for partitioning is made based on the ncut cost function. A continuous domain solution that is globally optimum is obtained by the eigendecomposition of the pairwise similarity matrix. The two techniques differ at the last step, where a discrete solution is obtained from the eigenvectors. Shi and Malik [4] used thresholding on a specific eigenvector, while $\mathrm{Ng}$ et al. [5] performed K-means clustering in the new space spanned by the eigenvectors that are associated with the first few eigenvalues.

$\mathrm{Yu}$ and Shi [7] later showed that to obtain a discrete solution, such heuristics are not needed, since the eigenvectors completely characterize the structure of all optimal solutions. The whole space of global optima can be navigated using orthogonal transforms. The near-global optimal solution can be obtained by iteratively solving for a discrete solution that is closest to a continuous global optima using an alternating optimization procedure. The method that uses this discretization step after obtaining the continuous solution is called multiclass spectral clustering (MSC) [7].

\section{A. Spectral Graph Partitioning Using Contour Information}

The main approaches to image segmentation are based on either regions or contours. Most region-based techniques perform sequential merging of segments based on an appropriate measure, which is often statistical. For example, a likelihood ratio test can be used to decide if two segments come from the same population or not. Region-based segmentation can also be performed by optimizing a global objective function, however in general, contour information is not utilized. On the other hand, contour-based techniques start with edge detection, followed by a linking process; edges are used to define segment boundaries. An important drawback is that only local information is used for edge detection and decisions regarding the segment boundaries are made prematurely. Leung and Malik [8] addressed this issue by computing contour information locally, but making the decision only after the information from the whole image is obtained. This approach allows the utilization of contour cue in a region-based setting [8], [9].

Information about the strength of a contour can be obtained through the orientation energy. Let $\mathcal{F}_{1}(x, y)$ be the second derivative of an elongated Gaussian kernel and $\mathcal{F}_{2}(x, y)$ be its Hilbert transform:

$$
\begin{aligned}
& \mathcal{F}_{1}(x, y)=\frac{d^{2}}{d y^{2}}\left(\frac{1}{C} \exp \left\{\frac{y^{2}}{\sigma^{2}}\right\} \exp \left\{\frac{x^{2}}{\lambda^{2} \sigma^{2}}\right\}\right) \\
& \mathcal{F}_{2}(x, y)=\operatorname{Hilbert}\left\{\mathcal{F}_{1}(x, y)\right\}
\end{aligned}
$$

where $C$ is a constant, $\sigma$ and $\lambda$ are the scale and elongation of the filter, respectively. For example, the orientation energy at angle $0^{\circ}$ has maximum response only for horizontal contours:

$$
\mathrm{OE}_{0^{\circ}}=\left\{I * \mathcal{F}_{1}\right\}^{2}+\left\{I * \mathcal{F}_{2}\right\}^{2}
$$

Rotated copies of the two filter kernels (shown in Figure 1) are able to pick up the edge contrast at different orientations.

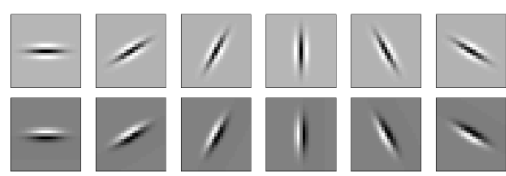

Fig. 1. Second derivative of an elongated Gaussian kernel (4) (top row) and its Hilbert transform (5) at different orientations (bottom row) [8].

At each pixel, the orientation energy, $\mathrm{OE}(x, y)$ is the maximum value of orientation energies calculated at a number of orientations, $\phi$ :

$$
\mathrm{OE}(x, y)=\max _{\phi} \mathrm{OE}_{\phi}(x, y)
$$

The orientation energy defined above has the following useful properties: (i) The second derivative of the Gaussian and its Hilbert transform form a quadrature pair. (ii) The filters are elongated, so that information is integrated along the edge. Thus, extended contours will stand out even if the contrast is low, as opposed to short contours with high contrast.

Based on the contour information, the dissimilarity of two pixels can be defined as the maximum value of the orientation energy that is encountered along the line joining those two pixels. Intuitively, if there is an extended contour (e.g., a field boundary) crossing between the two pixels, those pixels should belong to different partitions.

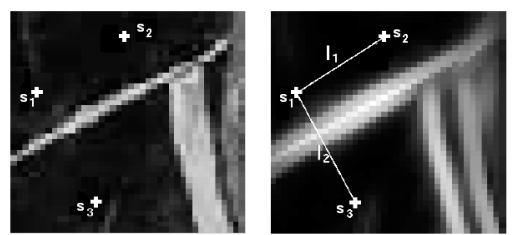

Fig. 2. Maximum value of orientation energy encountered along the line drawn between two pixels is a measure of dissimilarity. Adopted from [8]. 
Figure 2 illustrates this concept where $s_{1}, s_{2}$ and $s_{3}$ have similar intensity values. Typically in region-based segmentation, intensity is the only information source. Since these pixels have almost same intensity values, their similarities are high. However, there is an extended contour separating $s_{3}$ from $s_{1}$ and $s_{2}$. Thus, we expect $s_{1}$ to be much more strongly related to $s_{2}$ than to $s_{3}$. This intuition carries over to the definition of similarity based on contour information: If the maximum value of orientation energy on a line joining two pixels is high - suggesting the presence of an extended contour - the similarity of that pair should be low.

Formally, the dissimilarity of two pixels based on contour information, $\mathrm{d}_{\mathrm{c}}\left(s_{i}, s_{j}\right)$, is defined as:

$$
\begin{aligned}
\mathrm{d}_{\mathrm{c}}\left(s_{i}, s_{j}\right) & =\operatorname{OE}(\hat{x}) \\
\hat{x} & =\arg \max _{x \in l} \mathrm{OE}(x)
\end{aligned}
$$

where $l$ is the line joining $s_{i}$ and $s_{j}$; and $\hat{x}$ is the location where the orientation energy is maximum along $l$. Based on the contour information, the pairwise affinity is:

$$
W_{i j}^{C}=\exp \left\{\frac{-d_{c}^{2}\left(s_{i}, s_{j}\right)}{2 \sigma_{c}^{2}}\right\}
$$

where $\sigma_{c}$ is the scaling parameter for the kernel.

\section{Proposed Scheme For Segmentation of POLSAR DATA USING CONTOUR INFORMATION}

We propose the following scheme for segmenting Polarimetric SAR data using contour information:

1) Perform multi-looking on the single look complex (SLC) data set.

2) Form an affinity matrix for each of the channel powers (i.e., $|H H|^{2},|H V|^{2},|V V|^{2}$ ) using (10). Limit the number of affinity calculations per pixel by choosing an appropriate neighborhood size, representing proximity in the image plane; outside this neighborhood the similarity due to proximity will be "zero".

3) Form the combined affinity matrix, $W^{\text {tot }}$, using:

$$
W_{i j}^{t o t}=\prod_{d=1}^{n_{d}} W_{i j}^{C_{d}}
$$

where the subscript $d$ is the index for input data channels, and $n_{d}$ is the number of data channels.

4) Perform the multiclass spectral clustering algorithm [7] on $W^{\text {tot }}$. The following steps are taken:

- Calculate diagonal matrix $D$, where $D_{i i}=\sum_{j} W_{i j}$

- Find a continuous solution that is global optimum using eigendecomposition and normalization.

- Iteratively, solve for the discrete near-global optimum that is closest to the continuous solution. This is performed by alternating between the following steps until convergence: (a) Find the continuous optima closest to the discrete solution, (b) Find a discrete solution closest to the continuous optima.

\section{A. Implementation Details}

To calculate the orientation energy at a specific angle (6), the image is convolved with odd-symmetric and even-symmetric filters that are obtained using the difference of offset Gaussian (DOOG) kernel (For details see [10]). The parameters to select are: filter size, number of orientations, elongation $(\lambda)$ and scale $(\sigma)$ of the filter.

To compute the affinity matrix for each channel, we need to select the scale of the kernel $\left(\sigma_{c}\right)$. This choice can be made adaptively based on the maximum value of $\mathrm{OE}$ in the image. It is also required to choose the size of the neighborhood, outside which the weights will be zero.

\section{RESULTS AND DisCUSSION}

The results presented in this section are obtained using subsets of the polarimetric SAR scene shown in Figure 3(a). This C-band data set was acquired on 30 September 2004 by the Canadian Convair-580 aircraft. The scene covers the agricultural fields on Westham Island, located to the south of Vancouver. The area contains fields of corn, potatoes, variety of berries, hay, bare soil, some barley, wheat, pumpkin, turnip, red cabbage, broccoli, and grass. For our present purpose, we use manual segmentation as a reference solution.

Two test regions outlined in Figure 3(a) are expanded in Figures 3(b) and 3(e). Corresponding manual segmentations are given in Figures 3(c) and 3(f). Wishart classifier results that were obtained after speckle filtering [11] are shown in Figures $3(\mathrm{~d})$ and $3(\mathrm{~g})$. Using the manual segmentations as reference, Wishart classifier results are not as homogenous as we would like them to be. Assuming each segment to belong to a different class, classification accuracy is calculated based on the manual segmentation. For Test Regions \# 1 and \# 2, $81.6 \%$ and $74.1 \%$ of the pixels are assigned correctly.

Figures 4 and 5 present some results obtained with our new approach. Three polarimetric channel powers (i.e., $|H H|^{2}$, $\left.|H V|^{2},|V V|^{2}\right)$, RGB color composite formed using these channels, their corresponding orientation energy maps, and manual segmentations are also shown.

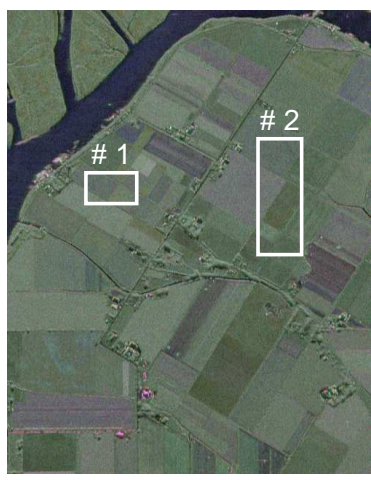

(a)

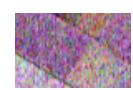

(b)

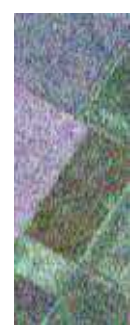

(e)

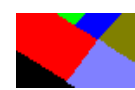

(c)

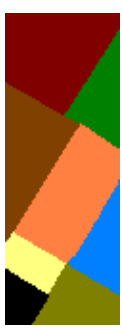

(f)

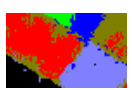

(d)

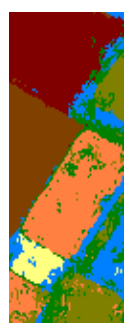

(g)
Fig. 3. Westham Island scene acquired by the Convair-580 (C) CSA 2004. (a) RGB color composite [HH-HV-VV] (b,e) RGB color composite - Test Regions \# 1 and \# 2 (c,f) Manual segmentations (d,g) Wishart classifier result 


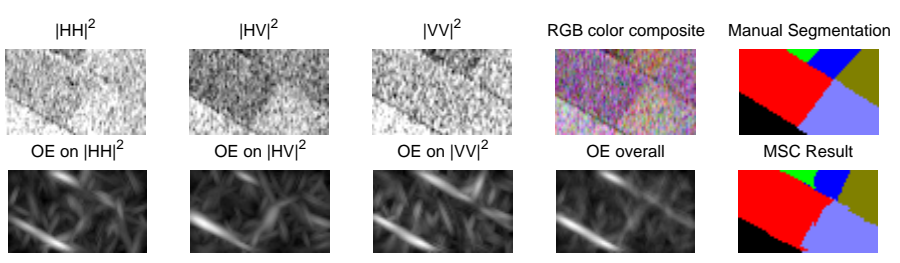

Fig. 4. Westham Island - Test Region \# 1 - Segmentation result of the proposed scheme based on MSC. $\left(\lambda^{2}=9\right.$, number of orientations $\left.=4\right)$

In Test Region \# 1, Figure 4, there are 6 different fields. The result of the proposed scheme (MSC) is very similar to our reference obtained by manual segmentation. On the other hand, Test Region \# 2, Figure 5, has 8 fields and the "MSC Result" in this case is not as accurate in finding the field boundaries, but still agrees with the reference segmentation in general. It is also more homogeneous than the Wishart result. Assuming each segment to represent a different class, the classification accuracies in terms of pixel count can be calculated for the two test regions as $94.3 \%$ and $94.7 \%$. These two examples demonstrate that the proposed scheme can obtain good segmentation results, both qualitatively (i.e., visual comparison) and quantitatively.

Results presented in Figures 4 and 5 were obtained using: Filter size $=21, \lambda^{2}=9$, number of orientations $=4$ and $\sigma^{2}=1$. To assess the effects of elongation and number of orientations, different values given in Table I were used. Although these parameters affect the orientation energy maps, they all seem to provide a similar level of accuracy.

These results were obtained using the contour information extracted from the three polarimetric channel powers. However, spectral graph partitioning allows us to include additional cues, such as patch-based similarity which was earlier shown to be promising [6]. Future work involves combining patchbased similarity and contour cues, performing detailed analysis using multiple data sets.

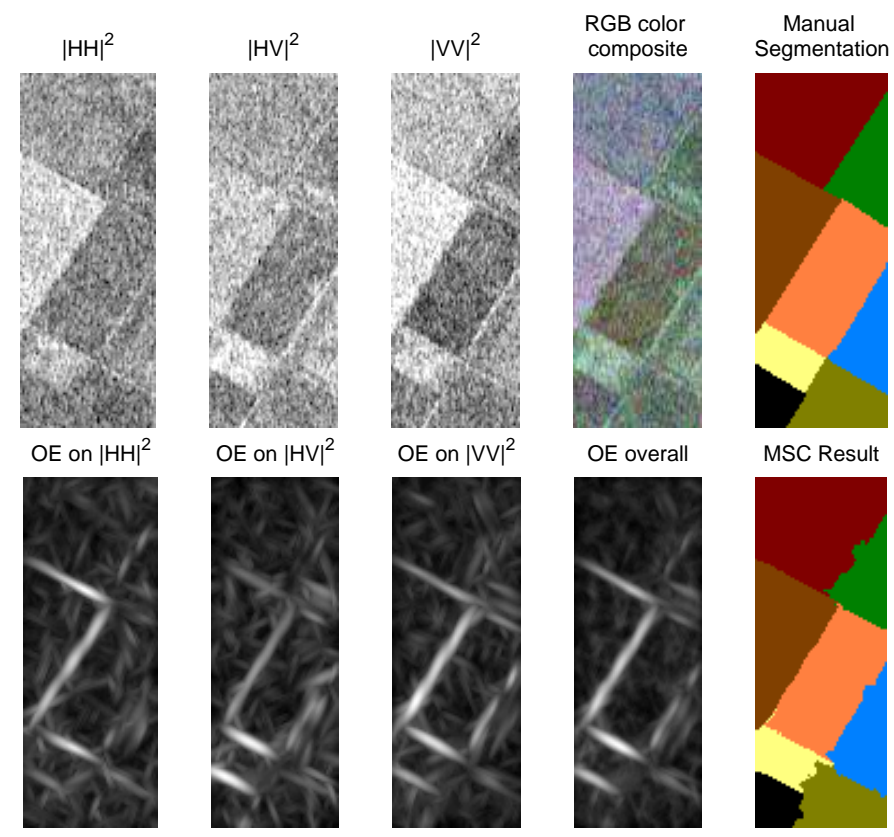

Fig. 5. Westham Island - Test Region \# $2 \quad\left(\lambda^{2}=9\right.$, \# of orientations $\left.=4\right)$
TABLE I

OVERALL CLASSIFICATION ACCURACY USING THE PROPOSED SCHEME

\begin{tabular}{c|c|c|c|c|c}
\hline \# of orientations & \multicolumn{2}{|c|}{4} & \multicolumn{2}{c|}{6} & \\
\hline Elongation $\left(\lambda^{2}\right)$ & 5 & 9 & 5 & 9 & \\
\hline \hline Test Region \# 1 & 94.3 & 94.3 & 94.2 & 89.6 & \\
\hline Test Region \# 2 & 95.2 & 94.7 & 94.5 & 94.4 & \\
\hline
\end{tabular}

\section{CONClusion}

A new method for polarimetric SAR data segmentation is proposed, based on spectral graph partitioning. Contour information is utilized by defining the pairwise similarities using the orientation energy. Multiclass spectral clustering is used to obtain a discrete solution that is close to the global optimum of our objective function (i.e., normalized cut). Preliminary results are obtained using C-band data acquired by an airborne system. The proposed method provides perceptually plausible segmentations - the results closely agree with the manual segmentations and they are more homogenous than those obtained using the Wishart classifier.

\section{ACKNOWLEDGMENT}

The authors acknowledge research funding from NSERC. The source of the data is the Canadian Space Agency (CSA) with the cooperation of Environment Canada, Natural Resources Canada (CCRS), Defense Research and Development Canada - Ottawa, and Radarsat International.

\section{REFERENCES}

[1] S. R. Cloude and E. Pottier, "An Entropy Based Classification Scheme for Land Applications of Polarimetric SAR," IEEE Transactions on Geoscience and Remote Sensing, vol. 35, no. 1, pp. 68-78, 1997.

[2] J. S. Lee, M. R. Grunes, T. L. Ainsworth, L. J. Du, D. L. Schuler and S. R. Cloude, "Unsupervised Classification Using Polarimetric Decomposition and the Complex Wishart Classifier," IEEE Transactions on Geoscience and Remote Sensing, vol. 37, no. 5, pp. 2249-2258, 1999.

[3] J. S. Lee, M. R. Grunes, E. Pottier and L. Ferro-Famil, "Unsupervised Terrain Classification Preserving Polarimetric Scattering Characteristics," IEEE Transactions on Geoscience and Remote Sensing, vol. 42, no. 4, pp. 722-731, 2004.

[4] J. Shi and J. Malik, "Normalized Cuts and Image Segmentation," IEEE Transactions on Pattern Analysis and Machine Intelligence, vol. 22, no. 8, pp. 888-905, 2000.

[5] A. Ng, M. Jordan and J. Malik, "On Spectral Clustering: Analysis and an Algorithm," in Advances in Neural Information Processing Systems (NIPS), 2001.

[6] K. Ersahin, I. G. Cumming and M. J. Yedlin, "Classification of Polarimetric SAR Data Using Spectral Graph Partitioning," in Proc. IEEE Int. Geoscience and Remote Sensing Symp., IGARSS'06, (Denver, USA), August 2006.

[7] S. X. Yu and J. Shi, "Multiclass Spectral Clustering," in International Conference on Computer Vision, (Nice, France), 11-17 Oct. 2003.

[8] T. Leung and J. Malik, "Contour Continuity in Region Based Image Segmentation," in Fifth European Conference on Computer Vision, (Freiburg, Germany), 1998.

[9] J. Malik, S. Belongie, T. Leung and J. Shi, "Contour and Texture Analysis for Image Segmentation," Int. J. of Computer Vision, vol. 43, no. 1, pp. 7-27, 2001.

[10] J. Malik and P. Perona, "Preattentive texture discrimination with early vision mechanisms," J. Optical Society of America, vol. 7, no. 2, pp. 923-932, 1990.

[11] J. S. Lee, M. R. Grunes and G. de Grandi, "Polarimetric SAR Speckle Filtering and Its Implications for Classification," IEEE Transactions on Geoscience and Remote Sensing, vol. 37, no. 5, pp. 2363-2373, 1999. 